\title{
THE EQUATION OF EVOLUTION IN A BANACH SPACE
}

\author{
BY \\ JOANNE ELLIOTT( $\left.{ }^{1}\right)$
}

1. Introduction. For each $t$ in some finite interval $[a, b]$, let $A(t)$ be a linear, not necessarily bounded, operator on a fixed Banach space $X$. We shall be concerned here with the construction of a family $\left\{U_{t, s}\right\}, a \leqq s \leqq t \leqq b$, of bounded linear transformations from $X$ to $X$ satisfying the equation

$$
D_{t} U_{t, 8} x=A(t) U_{t, 8} x \quad(x \in X)
$$

under the condition $U_{s, 8} x=x$. Equation (1.1) is the homogeneous equation of evolution. Rather stringent conditions on $A(t)$ are necessary in order for (1.1) to hold in the strictest sense. We therefore consider separately the left and right derivatives of $U_{t, s}$ with respect to $t$ and prove their existence only in a weak sense. Furthermore, in the general case we do not even require that $D_{t}^{ \pm} U_{t, 8} x$ be in the original space $X$. In Theorem 3.1, we employ an extension $\bar{A}$ of $A$ which may have values in a larger space.

In [3], T. Kato proved the existence of the family $\left\{U_{t, s}\right\}$ under certain regularity conditions on $A(t)$. Our method of construction differs considerably from Kato's; it is similar to that of Yosida [7] in the case $A(t)$ is independent of $t$. Our, and Kato's, first condition on $A(t)$ is condition $S$ of Definition 2.1. If $A(t)$ is independent of $t$, then this is the only condition needed, as Yosida proved in [7]. The operator $J_{\lambda}(t)$ defined by (2.2) converges strongly to $I$ as $\lambda \rightarrow \infty$, and the operator $A(t) J_{\lambda}(t)$ is a bounded operator for each $t \in[a, b]$ and each $\lambda>0$. We begin by studying (1.1), (or more generally, (2.12) and (2.13)), with $A(t)$ replaced by the bounded operator $A(t) J_{\lambda}(t)$ and obtain a solution $\left\{U_{t, s}^{\lambda}\right\}$ with $U_{s, s}^{\lambda}=I$. As $\lambda \rightarrow \infty, U_{t, s}^{\lambda}$ converges strongly to a limiting operator which gives the solution to the homogeneous equation of evolution, provided "solution" is suitably interpreted. The properties of the approximating family $\left\{U_{t, s}^{\lambda}\right\}$ are investigated in $\S 2$; the most general of our results is given in Theorem 3.1 of $\S 3$.

A comparison of Theorem 3.1 with Kato's Theorem 2 shows that neither result follows from the other. We impose conditions on both $A$ and $A^{*}$, whereas his is on $A$ alone. On the other hand, we obtain also the solution of the adjoint evolution equation. We relax slightly Kato's condition that the domain of $A(t)$ be independent of $t$, although our results in that direction are not general enough to permit application to two important special cases described below.

If $X$ is the space of continuous functions on some interval and $A(t)$ is a

Received by the editors August 8, 1960 and, in revised form, July 6, 1961.

(1) The author is grateful to the referee for his helpful criticisms, which led to a revision of this paper. 
second order linear differential operator for each $t \in[a, b]$, then the equation of evolution is a parabolic partial differential equation. Kato's theorem, and ours, can be applied to such equations, even in the case that the coefficients have singularities, cf. [6] where Kato's theorem is applied to singular operators of the form

$$
A(t)=D_{\mu(t)} D_{x(t)}+c(\cdot, t) I
$$

where, for each $t \in[a, b], \mu(t)$ is a measure, $x(t)$ a monotone function, and $c(\cdot, t)$ a real-valued function. There are, however, interesting special cases of (1.2) which cannot be handled by our theorems. We shall give two illustrations. First, the parabolic equation

$$
U_{t}=U_{x x} \quad(0 \leqq x<\infty)
$$

under the lateral condition $U_{x}(0, t)=p(t) U(0, t)$, where $p$ is a given function of $t$, and the initial condition $U(x, 0)=g(x)$, with $g$ a given element of $C[0, \infty]$. Here, we take $X=C[0, \infty] ; A(t) f$ is the restriction of $f^{\prime \prime}$ to functions with continuous second derivatives and satisfying $f^{\prime}(0)=p(t) f(0)$. A second example is

$$
U_{t}=D_{\mu(t)} D_{x} U,
$$

where $\mu(t)$ gives the point $x=t$ measure 1 and is the Lebesgue measure on Borel sets not containing $x=1$. The lateral condition is $U(0, t)=0$ and the initial condition is $U(x, 0)=f(x)$ with $f$ given in $C[0, \infty]$. In the first example, the dependence of the domain of $A(t)$ comes from the lateral condition; in the second example, the lateral condition is independent of $t$, but the measure $\mu$ appearing in the equation depends on $t$. In neither case does the operator $A(t)$ satisfy the entire set of conditions in Theorem 3.1.

For other references on the equation of evolution, see [1 (in Hilbert space);

2. Preliminaries. In this section we state several definitions and lemmas to be used in proving the results of $\S 3$.

Definition 2.1. An operator $A$ on a Banach space $X$ is said to have property $S$ if (1) it is a closed linear operator with domain dense in $X ;$ (2) the resolvent set of $A$ contains all positive reals, and

$$
\left\|(\lambda I-A)^{-1}\right\| \leqq \lambda^{-1} \quad(\lambda>0) .
$$

For convenience, we define

$$
J_{\lambda}=\lambda(\lambda I-A)^{-1} \quad(\lambda>0) .
$$

Following R. S. Phillips, we make the definition:

Definition 2.2. If $A$ has property $S$ on $X$, let $X_{\lambda}^{0}$ be the subspace of $X^{*}$ defined by $X^{0}=$ closure range $J_{\lambda}^{*}=$ closure domain $A^{*}$. Define $J^{0}=J_{\lambda^{*}} / X^{0}$ and $A^{0}=A^{*} / \operatorname{rng} J_{\lambda}^{0}$.

LEмma 2.1. The range of $J_{\lambda}^{0}$ is independent of $\lambda$ and $J_{\lambda}^{0} \rightarrow I$ strongly in $X^{0}$ 
as $\lambda \rightarrow \infty$; the operator $A^{0}$ has property $S$ in $X^{0}$ and $J_{\lambda}^{0}=\lambda\left(\lambda I-A^{0}\right)^{-1}$. $A$ norm $\|\cdot\|^{\prime}$ equivalent to $\|\cdot\|$ in $X$ is defined by

$$
\|x\|^{\prime}=\sup \left[\|y\| \leqq 1, y \in X^{0} ;|y(x)|\right] \text {. }
$$

Proof. For a proof of this lemma and for more details about $X^{0}$ and $A^{0}$, we refer to [2, Chapter XIV].

Definition 2.3. For each $t$ in some finite interval $[a, b]$, let $A(t)$ have property $S$ on a fixed Banach space $X$. Then $A(t)$ is said to have property $L$ on $[a, b]$ if, for each $\lambda>0$, str $\lim _{h \downarrow 0} J_{\lambda}(t+h)$ exists for $t \in[a, b)$, and $\operatorname{str} \lim _{h \downarrow 0} J_{\lambda}(t-h)$ exists for $t \in(a, b]$.

We use the notation:

$$
\operatorname{str} \lim _{h \downarrow 0} J_{\lambda}(t \pm h)=J_{\lambda}(t \pm 0)
$$

and

$$
\text { str } \lim _{h \downarrow 0} A(t \pm h) J_{\lambda}(t \pm h)=A J_{\lambda}(t \pm 0) .
$$

The limit in (2.5) exists if that in (2.4) does because of the identity

$$
A J_{\lambda}=\lambda\left(J_{\lambda}-I\right) \text {. }
$$

The next lemma concerns the construction of the approximating family $\left\{U_{t, \beta}^{\lambda}\right\}$ mentioned in $\$ 1$.

LEMMA 2.2. Let $A(t)$ have property $S$ for each $t$ in a finite interval $[a, b]$, on a fixed Banach space $X$. If, in addition, $A(t)$ has property $L$ on $[a, b]$, then the family of operators $\left\{U_{t, s}^{\lambda}\right\}, t$ and $s$ in $[a, b]$, given by

$$
U_{t, 8}^{\lambda} x=e^{-\lambda(t-s)} \sum_{n=0}^{\infty} \lambda^{n} I_{n}^{\lambda}(t, s) x
$$

with

$$
I_{0}^{\lambda}(t, s)=I
$$

and $I_{n}^{\lambda}$ defined by the Bochner integral

$$
\begin{aligned}
I_{n}^{\lambda}(t, s) x & =\int_{0}^{t} J_{\lambda}(u) I_{n-1}^{\lambda}(u, s) x d u \\
& =\int_{0}^{t} I_{n-1}^{\lambda}(t, v) J_{\lambda}(v) x d v
\end{aligned}
$$

has the following properties:

$$
\left\|U_{t, 8}^{\lambda}\right\| \leqq 1 \quad(t \geqq s),
$$




$$
U_{\mathbf{s}, 8}^{\lambda}=I
$$

The strong right and left derivatives of $U_{t, s}^{\lambda}$ satisfy

$$
\begin{array}{ll}
{\overline{D_{t}^{+}}}_{U_{t, 8}^{\lambda} x}^{\lambda}=A J_{\lambda}(t+0) U_{t, 8}^{\lambda} x & (s \in[a, b], t \in[a, b)), \\
D_{t}^{-} U_{t, 8}^{\lambda} x=A J_{\lambda}(t-0) U_{t, 8}^{\lambda} x & (s \in[a, b], t \in(a, b)], \\
D_{\imath}^{+} U_{t, 8}^{\lambda} x=-U_{t, 8}^{\lambda} A J_{\lambda}(s+0) x & (s \in[a, b), t \in[a, b]), \\
D_{,}^{-} U_{t, 8}^{\lambda} x=-U_{t, 8}^{\lambda} A J_{\lambda}(s-0) x & (s \in(a, b], t \in[a, b]),
\end{array}
$$

for all $x \in X$; finally, we have the composition rule

$$
U_{t, r}^{\lambda} U_{r, s}^{\lambda}=U_{t, 8}^{\lambda}
$$

for $r, s, t$ in $[a, b]$. For each $\lambda>0, U_{t, s}^{\lambda}$ is continuous in $(t, s)$ in the uniform norm and is the only family, strongly continuous in $(t, s)$, satisfying (2.10)(2.15).

Proof. From (2.1) and (2.2) it follows that

$$
\left\|I_{n}^{\lambda}(t, s)\right\| \leqq|t-s|^{n} / n !
$$

The series on the right of (2.7) therefore converges in the uniform norm and satisfies (2.10).

We next prove the equality of the two integrals in (2.9) by induction. The statement is certainly true for $n=1$. Now,

$$
\begin{aligned}
\int_{0}^{t} J_{\lambda}(u)\left\{\int_{0}^{u} I_{n-1}^{\lambda}(u, v) J_{\lambda}(v) x d v\right\} d u \\
=\int_{\varepsilon}^{t}\left\{\int_{v}^{t} J_{\lambda}(u) I_{n-1}^{\lambda}(u, v) J_{\lambda}(v) x d u\right\} d v .
\end{aligned}
$$

Thus, if (2.9) is true for some $n$, it is true for $n+1$.

We may thus write

$$
\begin{aligned}
U_{t, 8}^{\lambda} x & =e^{-\lambda(t-s)} x+e^{-\lambda(t-s)} \int_{s}^{t} \sum_{n=1}^{\infty} \lambda^{n} J_{\lambda}(u) I_{n-1}^{\lambda}(u, s) x d u \\
& =e^{-\lambda(t-s)} \int_{0}^{t} \sum_{n=1}^{\infty} \lambda^{n} I_{n-1}^{\lambda}(t, v) J_{\lambda}(v) x d v
\end{aligned}
$$

since the series inside the integrals converges in the uniform norm, uniformly in $u$ and $v$, respectively.

Upon differentiating (2.19) and using (2.6), we obtain (2.12)-(2.15). To prove $(2.16)$, we note that 


$$
D_{r}^{+} U_{t, r}^{\lambda} U_{r, 8}^{\lambda} x=\theta
$$

for $r \in[a, b)$. Since $U_{t, r}^{\lambda} U_{r, s}^{\lambda} x$ is continuous for $r \in[a, b]$, it must be constant, and setting $r=s$ we get (2.16).

Finally, to prove rhe uniqueness statement, suppose that there existed a second family $\left\{W_{t, s}^{\lambda}\right\}$, strongly continuous in $(s, t)$ satisfying (2.10)-(2.15). By the same argument used in proving (2.16), we can show that

$$
U_{t, r}^{\lambda} W_{r, 8}^{\lambda} x=U_{t, 8}^{\lambda} x=W_{t, 8}^{\lambda} x .
$$

This completes the proof of the theorem.

We next turn to the operator on $X^{*}$ adjoint to $U_{i, s}^{\lambda}$. We shall not make use of the full adjoint, but rather of the following contraction:

DeFinition 2.4. We define

$$
V_{B, t}^{\lambda}=\left(U_{t, 8}^{\lambda}\right) * / X_{t}^{0},
$$

where $s, t \in[a, b]$, and $X_{t}^{0}=$ closure range $J_{\lambda}^{*}(t)$.

Lemma 2.3. Let $A(t)$ satisfy the conditions of Lemma 2.2. Then $A^{0}(t)$ has property $S$ on $X_{t}^{0}$. If, in addition, $X_{t}^{0}=X^{0}$ is independent of $t$ and $A^{0}(t)$ has property $L$ on $[a, b]$, then the family of operators $\left\{V_{s, t}^{\lambda}\right\}$ defined in Definition 2.4 is a family of bounded linear transformations from $X^{0}$ to $X^{0}$ with the properties:

$$
\begin{array}{rlr}
\left\|V_{s, t}^{\lambda}\right\| \leqq 1 & (s \leqq t), \\
V_{s, s}=I & (s \in[a, b]), \\
D_{t}^{+} V_{s, t}^{\lambda} y=V_{s, t}^{\lambda} A J_{\lambda}^{0}(t+0) y\left({ }^{2}\right) & (s \in[a, b], t \in[a, b)), \\
D_{t}^{-} V_{s, t}^{\lambda} y=V_{s, t}^{\lambda} A J_{\lambda}^{0}(t-0) y & (s \in[a, b], t \in(a, b]), \\
D_{s}^{+} V_{s, t}^{\lambda} y=-V_{s, t}^{\lambda} A J_{\lambda}^{0}(s+0) y & (s \in[a, b), t \in[a, b]), \\
D_{s}^{-} V_{s, t}^{\lambda} y=-V_{s, t}^{\lambda} A J_{\lambda}^{0}(s-0) y & (s \in(a, b], t \in[a, b]),
\end{array}
$$

for all $y \in X^{0} ;$ and

$$
V_{s, r}^{\lambda} V_{r, t}^{\lambda}=V_{s, t}^{\lambda}
$$

Furthermore, $V_{s, t}^{\lambda}$ is continuous in $(s, t)$ in the uniform norm, and is the only family, strongly continuous in $(s, t)$, satisfying (2.23)-(2.28).

Proof. Referring back to the definition of $U_{t, s}^{\lambda}$ in (2.7)-(2.9), we see that

$$
\left[U_{t, 8}^{\lambda}\right]^{*} y=e^{-\lambda(t-s)} \sum_{n=0}^{\infty} \lambda^{n}\left[I_{n}^{\lambda}(t, s)\right]^{*} y,
$$

(2) For simplicity in writing, we shall use the notation: $A J_{\lambda}^{0}(t)=\left\{A J_{\lambda}(t)\right\}^{0}$. 
where

$$
\left[I_{n}^{\lambda}(t, s)\right]^{*} y=\int_{s}^{t} \int_{s}^{t_{n}} \cdots \int_{s}^{t_{2}} J_{\lambda}^{*}\left(t_{1}\right) \cdots J_{\lambda}^{*}\left(t_{n}\right) y d t_{1} \cdots d t_{n} .
$$

If $y \in X^{0}$, then $J_{\lambda}^{*}\left(t_{i}\right)$ can be replaced by $J_{\lambda}^{0}\left(t_{i}\right)$ in (2.31). The statements of the lemma either follow directly from the definition of $V_{s, t}^{\lambda}$ or are proved by the same sort of arguments as in Lemma 2.2.

Corollary 2.1. If $U_{t, s}^{\lambda}$ and $V_{s, t}^{\lambda}$ are defined as in Lemmas 2.2 and 2.3, then

$$
U_{t, 8}^{\lambda} x-x=\int_{8}^{t} A J_{\lambda}(r) U_{r, 8}^{\lambda} x d r=\int_{0}^{t} U_{t, r}^{\lambda} A J_{\lambda}(r) x d r,
$$

and

$$
V_{s, t}^{\lambda} y-y=\int_{s}^{t} V_{s, t}^{\lambda} A J_{\lambda}^{0}(r) y d r=\int_{0}^{t} A J_{\lambda}^{0}(r) V_{r, t}^{\lambda} y d r .
$$

Proof. This follows from the strong continuity of $U_{l, s}^{\lambda}$ and $V_{s, t}^{\lambda}$ in $(s, t)$, (2.11), (2.24), and the fact that their right derivatives are the same as those of the integrals in (2.32) and (2.33).

We end this section with some definitions and lemmas concerning the extension $\bar{A}$ of $A$ mentioned in $\$ 1$.

DEFINITION 2.5. Let $A(t)$ have property $S$ for each $t$ in some finite interval $[a, b]$ on a fixed Banach space $X$, and let $X_{t}^{0}=X^{0}$ be independent of $t$. We define an operator $\bar{A}(t)$ with domain in $X$ and range in $\left[X^{0}\right]^{*}$ by

$$
\{z=\bar{A}(t) x\} \leftrightarrow \lim _{\lambda \rightarrow \infty}\left(y, z-A J_{\lambda}(t) x\right)=0
$$

for all $y \in X^{0}$.

Note that if $X$ is renormed with the equivalent norm of Lemma 2.1, then $X$ is a subspace of $\left[X^{0}\right]^{*}$.

Lemma 2.4. The operator $\bar{A}(t)$ defined in Definition 2.5 is an extension of $A(t)$ and a contraction of $\left[A^{0}(t)\right]^{*}$.

Proof. The first statement is obvious, since if $x \in \mathrm{dmn} A(t)$, then $A J_{\lambda}(t) x$ $\rightarrow A(t) x$ in the norm of $X$. To prove the second statement, suppose that $x \in \mathrm{dmn} \bar{A}(t) x$; then for $y \in \mathrm{dmn} A^{0}(t)$,

$$
(y, \bar{A}(t) x)=\lim _{\lambda \rightarrow \infty}\left(y, A J_{\lambda}(t) x\right)=\lim _{\lambda \rightarrow \infty}\left(A J_{\lambda}^{0}(t) y, x\right)=\left(A^{0}(t) y, x\right),
$$

since $A J_{\lambda}^{0}(t) y \rightarrow A^{0}(t) y$ in the norm of $X^{0}$.

Definition 2.6. We define

$$
\bar{A}(t+0) x=w k\left(X^{0}\right) \lim _{h \downarrow 0} \bar{A}(t+h) x
$$

whenever $x \in \mathrm{dmn} \bar{A}(r), r \geqq t$, and the limit exists. 
Lemma 2.5. Suppose $A(t)$ satisfies the conditions of Definition 2.5 and has property $L$ on $[a, b]$. If there exists a dense set $D^{0} \subset X^{0}$ such that

$$
\left\|A J_{\lambda}^{0}(r) y\right\| \leqq M_{y},
$$$$
\left(r \geqq t, y \in D^{0}\right)
$$

where $M_{y}$ is a constant depending only on $y$, then for each $x \in X$ satisfying

$$
\left\|A J_{\lambda}(r) x\right\| \leqq M_{x}
$$

with $M_{x}$ a constant depending only on $x$, we have $x \in \operatorname{dmn} \bar{A}(t+0)$ and

$$
\bar{A}(t+0) x=\mathrm{wk}\left(X^{0}\right) \lim _{\lambda \rightarrow \infty} A J_{\lambda}(t+0) x .
$$

Proof. If $y \in D^{0}$, we have

$$
\left|\left(y,\left\{A J_{\lambda}(r)-A J_{\mu}(r)\right\} x\right)\right| \leqq\left|\lambda^{-1}-\mu^{-1}\right| \cdot\left|\left(A J_{\lambda}^{0}(r) y, A J_{\mu}(r) x\right)\right|,
$$

using the identity

$$
J_{\lambda}-J_{\mu}=\left(\lambda^{-1}-\mu^{-1}\right) A J_{\lambda} J_{\mu}
$$

Therefore, for $y \in D^{0}$,

$$
\left|\left(y,\left\{A J_{\lambda}(r)-A J_{\mu}(r)\right\} x\right)\right| \leqq\left|\lambda^{-1}-\mu^{-1}\right| \cdot M_{x} \cdot M_{y},
$$

by (2.40), (2.37), and (2.38). From (2.38) and the density of $D^{0}$ in $X^{0}$, we conclude that $x \in \mathrm{dmn} \bar{A}(r)$ for $r \geqq t$. For each $y \in D^{0}$, the convergence of $\left(y, A J_{\lambda}(r) x\right)$ is uniform in $r$ for $r \geqq t$ by (2.42). Hence, for $y \in D^{0}$, $\lim _{h \downarrow 0}(y, \bar{A}(t+h) x)$ exists and

$$
\lim _{h \downarrow 0}(y, \bar{A}(t+h) x)=\lim _{\lambda \rightarrow \infty}\left(y, A J_{\lambda}(t+0) x\right) .
$$

Now condition (2.38) implies that

$$
\left\|A J_{\lambda}(t+0) x\right\| \leqq M_{x}
$$

and

$$
\|\bar{A}(r) x\| \leqq M_{x},
$$$$
(r \geqq t) \text {. }
$$

Thus (2.39) follows from (2.43)-(2.45) and the density of $D^{0}$ in $X^{0}$.

3. The theorems. In this section, we prove our most general results about the homogeneous equation of evolution.

Theorem 3.1 (Existence). For each $t$ in some finite interval $[a, b]$, let $A(t)$ have property $S$ (Definition 2.1) on a fixed Banach space $X$, and property $L$ (Definition 2.3) on $[a, b]$; let $X^{0}=X_{t}^{0}$ (Definition 2.4) be independent of $t$, and let $A^{0}(t)$ have property $L$ on $[a, b]$. In addition, suppose that there exists a constant $C$, independent of $s, t, \lambda$, and $n$, such that for all $x \in \operatorname{dmn} A(s)$, 


$$
\left\|A(t) J_{\lambda}(t) J_{\lambda}\left(t_{n}\right) \cdots J_{\lambda}\left(t_{1}\right) x\right\| \leqq C\|A(s) x\|,
$$

and for all $y \in \mathrm{dmn} A^{0}(t)$,

$$
\left\|A^{0}(s) J_{\lambda}^{0}(s) J_{\lambda}^{0}\left(t_{1}\right) \cdots J_{\lambda}^{0}\left(t_{n}\right) y\right\| \leqq C\left\|A^{0}(t) y\right\|
$$

whenever $a \leqq s=t_{0} \leqq t_{1} \leqq \cdots \leqq t_{n+1}=t \leqq b$ is a partition of $[s, t]$.

Under these conditions there exists a family $\left\{U_{t, 8}\right\}, a \leqq s \leqq t \leqq b$, of linear transformations from $X$ to $X$, strongly continuous in $(t, s)$ for $t \geqq s$, and satisfying

$$
\begin{aligned}
\left\|U_{t, s}\right\| & \leqq 1, \\
U_{s, s} & =I, \\
U_{t, r} U_{r, s} & =U_{t, 8} \quad(s \leqq r \leqq t) .
\end{aligned}
$$

If $\bar{A}(t)$ and $\bar{A}(t+0)$ are the operators defined in Definitions 2.5 and 2.6, then

$$
U_{t, 8} x \in \mathrm{dmn} \bar{A}(r)
$$

and

$$
U_{t, s} \in \operatorname{dmn} \bar{A}(t+0)
$$

whenever $x \in \operatorname{dmn} A\left(s^{\prime}\right)$ for some $s^{\prime} \leqq s$. Finally, for $x \in \operatorname{dmn} A\left(s^{\prime}\right)$ we have

$$
D_{t}^{+} U_{t, 8} x=\bar{A}(t+0) U_{t, 8} x \quad(a \leqq s \leqq t<b)
$$

and

$$
\overline{D_{t}} U_{t, 8} x=\mathrm{wk}\left(X^{0}\right) \lim _{h \downarrow 0} \bar{A}(t-h) U_{t-h, 8} x, \quad(a \leqq s<t \leqq b)
$$

the derivatives existing in the weak $\left(X^{0}\right)$ sense in $\left(X^{0}\right)^{*}$.

The family $\left\{V_{s, t}\right\}, a \leqq s \leqq t \leqq b$, given by $V_{s, t}=U_{t, s}^{*} / X^{0}$ is a family of operators from $X^{0}$ to $X^{0}$ with the properties:

$$
\begin{aligned}
(3.10) & \left\|V_{s, t}\right\| & \leqq 1 & (s \leqq t), \\
(3.11) & V_{\imath, t} & =I, & \\
(3.12) & V_{c, r} V_{r, t} & =V_{s, t} & (s \leqq r \leqq t), \\
(3.13) & D_{\imath}^{-} V_{c, t} y & =-\operatorname{wk}(X) \lim _{h \downarrow 0} A^{*}(s-h) V_{s, t} y & (a<s \leqq t \leqq b),
\end{aligned}
$$

the derivatives in (3.13) and (3.14) existing in the weak $(X)$ sense.

Proof. Let us first state a few consequences of our conditions on $A(t)$. Conditions (3.1) and (3.2) imply, in particular, that 


$$
\left\|A J_{\lambda}(t) x\right\| \leqq C \cdot\|A(s) x\|
$$

whenever $x \in \operatorname{dmn} A(s)$, and that

$$
\left\|A J_{\lambda}^{0}(s) y\right\| \leqq C \cdot\left\|A^{0}(t) y\right\| \quad(t \geqq s)
$$

whenever $y \in \operatorname{dmn} A^{0}(t)$. Going back to the definitions of $U_{t, s}^{\lambda}$ in (2.7)-(2.9), and of $V_{s, t}^{\lambda}$ in (2.22), (2.30), and (2.31), we see that two more consequences of (3.1) and (3.2) are

$$
\left\|A J_{\lambda}(t) U_{t, e x}^{\lambda}\right\| \leqq C \cdot\left\|A\left(s^{\prime}\right) x\right\|,
$$

whenever $x \in \operatorname{dmn} A\left(s^{\prime}\right)$ for some $s^{\prime} \leqq s ;$ and

$$
\left\|A J_{\lambda}^{0}(s) V_{s, t}^{\lambda} y\right\| \leqq C \cdot\left\|A^{0}\left(t^{\prime}\right) y\right\|,
$$

whenever $y \in \operatorname{dmn} A^{0}\left(t^{\prime}\right)$ for some $t^{\prime} \geqq t$. Using the identity (2.41), we have for each $\mu>0, \lambda>0$

(3.19) $\left\|A J_{\mu}(t) U_{t, s}^{\lambda} x\right\| \leqq\left|\mu^{-1}-\lambda^{-1}\right| \cdot\left\|A J_{\mu} A J_{\lambda}(t) U_{t, 8}^{\lambda} x\right\|+\left\|A J_{\lambda}(t) U_{t, s}^{\lambda} x\right\|$.

Thus if $x \in \operatorname{dmn} A\left(s^{\prime}\right)$ for some $s^{\prime} \leqq s$, we can combine (3.19) with (3.17) to obtain

$$
\begin{aligned}
\left\|A J_{\mu}(t) U_{t, 8}^{\lambda} x\right\| & \leqq C\left\{2\left|1-\mu \cdot \lambda^{-1}\right|+1\right\} \cdot\left\|A\left(s^{\prime}\right) x\right\| \\
& \leqq C\left\{3+2 \mu \cdot \lambda^{-1}\right\}\left\|A\left(s^{\prime}\right) x\right\| .
\end{aligned}
$$

We have also used here the fact that

$$
\left\|A J_{\mu}(t) x\right\| \leqq 2 \mu
$$

from (2.6) and (2.1). Similarly, if $y \in \operatorname{dmn} A^{0}\left(t^{\prime}\right)$ for some $t^{\prime} \geqq t$, we have

$$
\left\|A J_{\mu}^{0}(t) V_{s, t}^{\lambda}\right\|\left\|\leqq C\left\{3+2 \mu \cdot \lambda^{-1}\right\} \cdot\right\| A\left(t^{\prime}\right) y \| .
$$

We now turn to the proof of the theorem. We shall show that as $\lambda \rightarrow \infty$, the operator $U_{t, s}^{\lambda}$ converges strongly to a limiting operator $U_{t, . .}$ For $\lambda, \mu>0$ we have,

$$
U_{t, r}^{\mu} U_{r^{\prime}, 0}^{\lambda} x-U_{t, 8}^{\mu} x=\int_{0}^{r^{\prime}} U_{t, r}^{\mu}\left\{A J_{\lambda}(r)-A J_{\mu}(r)\right\} U_{r, s}^{\lambda} x d r,
$$

since both sides are continuous in $r^{\prime}$ on $[a, b]$, have the same strong right derivatives in $r^{\prime}$, and agree when $r^{\prime}=s$. Putting $r^{\prime}=t$, we obtain 


$$
U_{t, 8}^{\lambda} x-U_{t, 8}^{\mu} x=\int_{0}^{t} U_{t, r}^{\mu}\left\{A J_{\lambda}(r)-A J_{\mu}(r)\right\} U_{r, 8}^{\lambda} x d r .
$$

By (2.41) this reduces to

$$
U_{t, 8}^{\lambda} x-U_{t, 8}^{\mu} x=\left(\lambda^{-1}-\mu^{-1}\right) \int_{s}^{t} U_{t, r}^{\mu} A J_{\mu}(r) A J_{\lambda}(r) U_{r, 8}^{\lambda} x d r .
$$

Now let $\nu$ be an arbitrary positive number. For each $y \in X^{0}$, we have for $t \geqq s$,

$$
\begin{aligned}
\left|\left(y, J_{\nu}(t)\left\{U_{t, 8}^{\lambda}-U_{t, 8}^{\mu}\right\} x\right)\right| \\
\quad \leqq\left|\lambda^{-1}-\mu^{-1}\right| \cdot \int_{0}^{t}\left|\left(A J_{\mu}^{0}(r) V_{r, b}^{\mu} J_{\nu}^{0}(t) y, A J_{\lambda}(r) U_{r, s}^{\lambda} x\right)\right| d r,
\end{aligned}
$$

using (3.25). Thus, by (3.17), (3.18), and (3.21), we have

$$
\begin{aligned}
\left|\left(y, J_{\nu}(t)\left\{U_{t, 8}^{\lambda}-U_{t, 8}^{\mu}\right\} x\right)\right| & \leqq\left|\lambda^{-1}-\mu^{-1}\right| \cdot C^{2} \cdot(t-s) \cdot\left\|A J_{\nu}^{0}(t) y\right\| \cdot\|A(s) x\| \\
& \leqq\left|\lambda^{-1}-\mu^{-1}\right| \cdot C^{2} \cdot(t-s) \cdot 2\|A(s) x\| \cdot \nu
\end{aligned}
$$

whenever $t \geqq s$, and $x \in \mathrm{dmn} A(s)$. In the notation of Lemma 2.1, we can conclude that

$$
\left\|J_{\nu}(t)\left\{U_{t, 8}^{\lambda}-U_{t, s}^{\mu}\right\} x\right\|^{\prime} \leqq 2\left|\lambda^{-1}-\mu^{-1}\right| \cdot C^{2} \cdot(t-s) \cdot\|A(s) x\| \cdot \nu .
$$

But since $\|\cdot\|^{\prime}$ is equivalent to $\|\cdot\|$, it follows that $J_{v}(t) U_{t, s}^{\lambda} x$ converges as $\lambda \rightarrow \infty$, in the norm of $X$, whenever $x \in \operatorname{dmn} A(s)$. This implies convergence for all $x \in X$, since $\operatorname{dmn} A(s)$ is dense in $X$, and $\left\|J_{\nu}(t) U_{t, s}^{\lambda}\right\| \leqq 1$. But

$$
U_{t, 8}^{\lambda}=J_{\nu}(t) U_{t, 8}^{\lambda} x+\nu^{-1} A J_{\nu}(t) U_{t, 8}^{\lambda} x,
$$

and by (3.20),

$$
\left\|\nu^{-1} A J_{\nu}(t) U_{t, x}^{\lambda}\right\| \leqq C\left(3 \nu^{-1}+2 \lambda^{-1}\right) \cdot\|A(s) x\|,
$$

whenever $x \in \mathrm{dmn} A(s) x$. It follows from the convergence of $J_{\nu}(t) U_{t, s}^{\lambda} x$, (3.29), and (3.30), that $U_{t, 0}^{\lambda} x$ converges in the norm of $X$ as $\lambda \rightarrow \infty$, for all $x \in X$.

It follows directly from the convergence of $U_{t, 0} x,(2.10),(2.11)$, and (2.16) that (3.3)-(3.5) hold. have

Next, we prove the strong continuity of $U_{t, s}^{\lambda}$ in $(t, s)$. From (2.32) we 


$$
\begin{aligned}
U_{t \pm h, 8 \pm k}^{\lambda} x-U_{t, 8}^{\lambda} x= & \int_{0}^{\bullet \pm k} U_{t \pm h, r}^{\lambda} A J_{\lambda}(r) x d r \\
& +\int_{t}^{t \pm h} A J_{\lambda}(r) U_{r, 8}^{\lambda} x d r .
\end{aligned}
$$

If $x \in \operatorname{dmn} A(s)$ and $t \pm h \geqq s \pm k$, we have from (3.15) and (3.17)

$$
\left\|U_{t \pm h, \varepsilon \pm k}^{\lambda} x-U_{t, 8}^{\lambda} x\right\| \leqq C(h+k) \cdot\|A(s) x\| \quad(h, k \geqq 0 ; t>s),
$$

and if $h \geqq k$,

$$
\left\|U_{*+h, 0+k}^{\lambda} x-x\right\| \leqq C(h+k) \cdot\|A(s) x\| \quad(h, k \geqq 0) .
$$

Letting $\lambda \rightarrow \infty$, we see that (3.32) and (3.33) also hold when $U_{t, s}^{\lambda}$ is replaced by $U_{t, \mathrm{~s}}$. Since $\mathrm{dmn} A(s)$ is dense in $X$, the strong continuity of $U_{t, \mathrm{e}}$ in $(t, s)$ for $t \geqq s$ follows.

We now establish (3.6) and (3.7), making use of Lemma 2.5. For any $y \in \operatorname{dmn} A^{0}(b)$, we have

$$
\left\|A J_{\lambda}^{0}(r) y\right\| \leqq C \cdot\left\|A^{0}(b) y\right\|
$$

by (3.16). Hence (2.37) is satisfied with $D^{0}=\operatorname{dmn} A^{0}(b)$. We next show that $U_{t, x} x \in \operatorname{dmn} \bar{A}(r)$ for $r \geqq t$, whenever $x \in \operatorname{dmn} A\left(s^{\prime}\right)$ for some $s^{\prime} \leqq s$. To accomplish this we must show that $A J_{\lambda}(r) U_{t, 8} x$ converges weakly $\left(X^{0}\right)$ as $\lambda \rightarrow \infty$. If $y \in \operatorname{dmn} A^{0}(r)$ then

$$
\lim _{\lambda \rightarrow \infty}\left(y, A J_{\lambda}(r) U_{t, 8} x\right)=\left(A^{0}(r) y, U_{t, 8} x\right) .
$$

Furthermore, interchanging $\lambda$ and $\mu$ in (3.20) and letting $\mu \rightarrow \infty$, we obtain

$$
\left\|A J_{\lambda}(t) U_{t, 8} x\right\| \leqq 3 C \cdot\left\|A\left(s^{\prime}\right) x\right\|,
$$

when $x \in \operatorname{dmn} A\left(s^{\prime}\right)$ for some $s^{\prime} \leqq s$. Since $\operatorname{dmn} A^{0}(r)$ is dense in $X^{0}$, it follows from (3.36) that the limit on the left of (3.35) must exist for all $y \in X^{0}$, provided $x \in \operatorname{dmn} A\left(s^{\prime}\right)$ for some $s^{\prime} \leqq s$. Now all the conditions of Lemma 2.5 are satisfied, with $D^{0}=\operatorname{dmn} A^{0}(b)$ and $x$ replaced by $U_{t, x} x, x \in \operatorname{dmn} A\left(s^{\prime}\right)$ for some $s^{\prime} \leqq s$. From this it follows that (3.7) holds. Arguments similar to those used in establishing Lemma 2.5 can be used to show that $\operatorname{wk}\left(X^{0}\right) \lim _{h \downarrow 0} \bar{A}(t-h) U_{t-h, e} x$ exists (in $\left.\left\{X^{0}\right\} *\right)$, for $x \in \operatorname{dmn} A\left(s^{\prime}\right), s^{\prime} \leqq s$.

To prove (3.8) and (3.9), we note that

$$
\left(y, U_{t, 8} x-x\right)=\int_{0}^{t}\left(y, \bar{A}(r) U_{r, 8} x\right) d r
$$


using (2.32) and letting $\lambda \rightarrow \infty$. With the results of the previous paragraph, we may differentiate (3.37) to get (3.8) and (3.9).

Statements (3.10)-(3.12) for $V_{\text {s.t }}$ follow directly from its definition. We also note that

$$
V_{e, t} y=\operatorname{wk}(X) \lim _{\lambda \rightarrow \infty} V_{s, t}^{\lambda} y \text {, }
$$

for $y \in X^{0}$. It is also easily shown, the arguments being similar to those we have used for $U_{t, s}$, that $\operatorname{wk}(X) \lim _{\lambda \rightarrow \infty} A J_{\lambda}^{0}(r) V_{s, t} y$ exists when $r \leqq t$, $y \in \operatorname{dmn} A^{0}\left(t^{\prime}\right)$ for some $t^{\prime} \geqq t$, and that $V_{\bullet, t} y \in \operatorname{dmn} A^{*}(r)$. We thus obtain an integral representation for $V_{s, t}$ from (2.33). The existence of the limits

$$
\operatorname{wk}(X) \lim _{h \downarrow 0} A^{*}(s-h) V_{\imath, t} y
$$

and

$$
\mathrm{wk}(X) \lim _{h \downarrow 0} A^{*}(s+h) V_{s+h, t} y
$$

follow from the fact that

$$
\lim _{\lambda \rightarrow \infty}\left(A J_{\lambda}^{0}(r) V_{e, t} y, x\right)=\left(A^{*}(r) V_{s, t} y, x\right),
$$

the convergence being uniform for $r \leqq s$, whenever $x \in \mathrm{dmn} A(a)$. These arguments are so similar to those we have used before for $U_{t, 8}$ that we do not reproduce the details.

ThEOREM 3.2 (UnIQueness). Under the hypotheses of Theorem 3.1, the family $\left\{U_{t, s}\right\}$ is the only family of bounded linear transformations from $X$ to $X$, strongly continuous in $t$ for fixed $s$, satisfying (3.4), (3.6)-(3.8), and the condition

$$
\left\|A J_{\lambda}(t) U_{t, 8} x\right\| \leqq K \cdot\left\|A\left(s^{\prime}\right) x\right\|
$$

whenever $x \in \operatorname{dmn} A\left(s^{\prime}\right)$, with $K$ a constant independent of $\lambda$ and $x$.

Proof. Note that we have proved (3.41) for the family $\left\{U_{t, 8}\right\}$ in (3.36). If there were a second such family $U_{i, 8}^{\prime}$ then by an argument similar to that used in proving (3.24), we would have for $y \in \operatorname{dmn} A^{0}(t), x \in \operatorname{dmn} A(s)$,

$$
\begin{aligned}
\left(y, U_{t, 8}^{\prime} x-U_{t, 8}^{\lambda} x\right) & =\int_{0}^{t}\left(V_{r, t}^{\lambda} y,\left\{\bar{A}(r)-A J_{\lambda}(r)\right\} U_{r, 8}^{\prime} x\right) d r \\
& =\lim _{\mu \rightarrow \infty} \int_{0}^{t}\left(V_{r, t}^{\lambda} y,\left\{A J_{\mu}(r)-A J_{\lambda}(r)\right\} U_{r, 8}^{\prime} x\right) d r .
\end{aligned}
$$


By (2.41), this gives

$$
\left(y, U_{t, 8}^{\prime} x-U_{t, 8}^{\lambda} x\right)=\lim _{\mu \rightarrow \infty}\left(\mu^{-1}-\lambda^{-1}\right) \int_{s}^{t}\left(A J_{\lambda}^{0}(r) V_{r, t}^{\lambda} y, A J_{\mu}(r) U_{r, 8}^{\prime} x\right) d r
$$

If $x \in \mathrm{dmn} A(s)$ and $y \in \mathrm{dmn} A^{0}(t)$, we can apply (3.41) and (3.18) to conclude

$$
\left|\left(A J_{\lambda}^{0}(r) V_{r, t}^{\lambda} y, A J_{\mu}(r) U_{r, 8}^{\prime} x\right)\right| \leqq M\left\|A^{0}(t) y\right\| \cdot\|A(s) x\|,
$$

where $M$ is a constant. But this implies that

$$
\lim _{\lambda \rightarrow \infty}\left(y, U_{t, 8}^{\prime} x-U_{t, 8}^{\lambda} x\right)=0=\left(y, U_{t, 8} x-U_{t, 8} x\right)
$$

for $y \in \operatorname{dmn} A^{0}(t), x \in \operatorname{dmn} A(s)$. Since $\operatorname{dmn} A^{0}(t)$ is dense in $X^{0}$, the expression on the right of (3.45) vanishes for all $y \in X^{0}$. Therefore by Definition 2.2 and Lemma 2.1, $U_{t, s}^{\prime} x=U_{t, s} x$ for all $x \in \operatorname{dmn} A(s)$. But $\operatorname{dmn} A(s)$ is dense in $X$ and the equality must hold for all $x \in X$.

Our final theorem is a restatement of Theorem 3.1 in the special case that $X$ is reflexive.

THEOREM 3.3. If, in addition to the hypotheses of Theorem 3.1, we assume that $X$ is reflexive, then $\operatorname{dmn} A(s) \subset \mathrm{dmn} A(t)$ for $s \leqq t$, and $\bar{A}(t)$ can be replaced by $A(t)$ in (3.6)-(3.9). In addition, $X^{0}=X^{*}$, and $A^{*}(t)=A^{0}(t)$ for $t \in[a, b]$.

Proof. If $X^{0} \neq X^{*}$, then there would exist a $z \in X, z \neq \theta$, such that $\left(J_{\lambda}^{*}(t) y, z\right)$ $=0$ for all $y \in X^{*}$. But this implies $J_{\lambda}(t) z=\theta$, which is impossible unless $z=\theta$. Thus $X^{0}=X$ and, by definition, $A^{0}(t)=A^{*}(t)$ for $t \in[a, b]$.

Next we show that $\bar{A}(t)$ can be replaced by $A(t)$. Suppose $x \in \mathrm{dmn} A(s)$; if $r \geqq t$, then $A J_{\lambda}(r) U_{t, 8} x$ converges weakly and, by (3.36), boundedly in norm as $\lambda \rightarrow \infty$. This implies (cf. [3, Lemma 5]) that $U_{t, 8} x \in \mathrm{dmn} A(r)$, and that $A J_{\lambda}(r)$ converges weakly to $A(r) U_{t, 8} x$. Therefore, if $r \geqq t$, then $\bar{A}(r) U_{t, 8} x$ $=A(r) U_{t, s} x$. Since $U_{s, s}=I$, this implies also that $\operatorname{dmn} A(s) \subset \operatorname{dmn} A(r)$ when $r \geqq s$.

\section{BIBLIOGRAPHY}

1. C. Foias, G. H. Gussi et V. Poenaru, L'étude de l'équation $d u / d \tau=A(\tau) u$ pour certaines classes d'opérateurs non bornés de l'espace de Hilbert, Trans. Amer. Math. Soc. 86 (1957), 335347.

2. E. Hille and R. S. Phillips, Functional analysis and semi-groups, Amer. Math. Soc. Collog. Publ. Vol. 31, Amer. Math. Soc., Providence, R. I., 1957.

3. T. Kato, Integration of the equation of evolution in a Banach space, J. Math. Soc. Japan, 5 (1953), 208-234.

4. R. S. Phillips, Perturbation theory for semi-groups of linear operators, Trans. Amer. Math. Soc. 74 (1953), 199-221. 
5. L. Schwartz, Les équations d'évolution liees au produit de composition, Ann. Inst. Fourier, Grenoble 2 (1950), 19-49.

6. D. G. S. Stockton, Singular parabolic differential equations with time dependent coeffcients, Brown University thesis, 1958.

7. K. Yosida, On the differentiability and the representation of one-parameter semi-groups of linear operators, J. Math. Soc. Japan 1 (1948), 15-21.

Barnard College, Columbia University, NEW YoRK, NEW YoRK 